\title{
ALVEOLAR RHABDOMYOSARCOMA OF SINONASAL TRACT
}

\section{Noreen Akhtar, Safana Sadaf, Sajid Mushtaq}

Department of Histopathology, Shaukat Khanum Memorial Cancer Hospital and Research Centre, Lahore, Pakistan

Received: 20 December 2016 / Accepted: 25 December 2016

A biopsy of a 27-year-old male was received with a history of bloody nasal discharge for the past 1 year and sinonasal growth. Tissue was composed of multiple pieces collectively measuring $3 \times 3 \mathrm{~cm}$ in aggregate. Histologically, tumour was composed of round blue cells arranged in nested pattern. The tumour cells showed scanty cytoplasm, moderate nuclear pleomorphism, hyperchromatic nuclei and indistinct nucleoli. Brisk mitotic activity was noted [Figure 1]. No spindled cells, myxoid change and necrosis were seen.

Immunohistochemically, the tumour cells showed strong cytoplasmic desmin positivity. Tumour cells showed negative expression of CK, P40, NSE, calretinin and synaptophysin. In situ hybridisation for Epstein-Barr virus-encoded RNA was also negative [Figure 1]. Final diagnosis was rendered as alveolar rhabdomyosarcoma.

The differential diagnosis of round blue cell tumours in head and neck region includes rhabdomyosarcoma, olfactory neuroblastoma, sinonasal carcinoma, Ewing's sarcoma, small cell carcinoma and lymphomas. In this case, tumour cells showed nested pattern and strong desmin positivity favouring alveolar rhabdomyosarcoma.

Rhabdomyosarcoma is a malignant tumour of mesenchymal tissue derivation. It represents most common soft tissue sarcoma of paediatric population. ${ }^{[1]}$ Amongst alveolar and embryonal rhabdomyosarcoma, alveolar most commonly occur in adolescents than young children. ${ }^{[2]}$

Approximately $40 \%$ of rhabdomyosarcoma involve head and neck region. Lesion in sinonasal tract commonly presents as symptoms of sinusitis, cranial nerve

Correspondence: Noreen Akhtar, Department of Pathology, Shaukat Khanum Memorial Cancer Hospital and Research Centre, Lahore, Pakistan.Email: nakhter@skm.org.pk palsies, proptosis and mass. ${ }^{[3]}$ Immunohistochemically, rhabdomyosarcoma shows positivity for desmin, myogenin, myoD1 and muscle-specific actin.

Histologically, alveolar rhabdomyosarcoma cells show cohesive nests of round cells, outlined by fibrous septa with picket row configuration. Alveolar rhabdomyosarcoma has reciprocal translocations which are absent in embryonal rhabdomyosarcomas.

These translocations, $\mathrm{t}(2 ; 13)(\mathrm{q} 35 ; \mathrm{q} 14)$ and $\mathrm{t}(1 ; 13)$ (p36;q14), generate the fusion genes PAX3- FOXO1 and PAX7-FOXO1, respectively. ${ }^{[2]}$ The treatment modalities for alveolar rhabdomyosarcoma include surgery, chemotherapy and radiotherapy. Prognosis depends on stage and histological type. As compared to embryonal rhabdomyosarcoma, alveolar type behave in more aggressive fashion. ${ }^{[4]}$

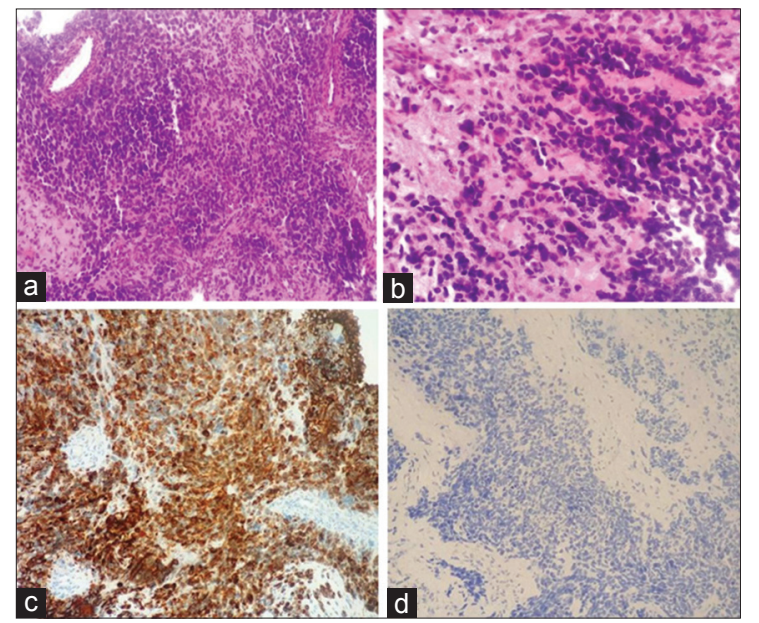

Figure 1: (a) Tumour arranged in nests, composed of round blue cells with moderate cytoplasm, hyperchromatic nuclei and indistinct nucleoli. (b) High-power view of tumour cells with nuclear pleomorphism and few rhabdoid cells. (c) Desmin strongly positive in tumour cells showing cytoplasmic staining pattern. (d) Tumour cells negative for CK, P40, calretinin, synaptophysin, NSE and EBER 


\section{Conflict of Interest}

The authors declare that they have no conflict of interest.

\section{References}

1. Wu JM, Montgomery E. Classification and pathology. Surg Clin North Am 2008;88:483-520, v-vi.

2. Soledad G, Toledo D, Sanchez J, et al. Molecular Genetics of
Rhabdomyosarcoma. Chichester: John Wiley and Sons Ltd.; 2012. Available from: http://www.els.net. [Last accessed 2016 Dec 01].

3. Legendre BW, Nelson BL. Alveolar rhabdomyosarcoma of the paranasal sinuses. Head Neck Pathol 2008;2:302-4.

4. Fletcher DM, Unni KK, Mertens F, editors. World Health Organization Classification of Tumours: Tumours of Soft Tissue and Bone. Lyon: IARC Press; 2002. 